the proposed ion beam technique. If the accelerating voltage is $V$ and the ion current $I$ then

$$
\begin{aligned}
& v=\text { const. } \sqrt{ } V \\
& \varepsilon=I V
\end{aligned}
$$

From equations (1-4)

$$
\begin{aligned}
& v=\text { const. }\left(1-t / t_{0}\right)^{-1} \\
& V=\text { const. }\left(1-t / t_{0}\right)^{-2} \\
& I=\varepsilon / V=\text { const. }\left(1-t / t_{0}\right)^{1 / 8}
\end{aligned}
$$

Since the ion current is approximately proportional to the laser light intensity, a simultaneous change in both the accelerating voltage and laser light intensity can produce the power profile equation (2).

The proposed technique offers two possibilities for nuclear micro-explosions:

First, the ignition of rather large micro-explosions with two beams of $\sim 100 \mathrm{MJ}$ produced in two $\sim 100 \mathrm{~m}$ drift tubes clashing head on, compressing and heating between them a sandwich of dense thermonuclear or fission-fusion hybrid material. For fusion pellets a minimum energy of $\sim 10^{2}-10^{3} \mathrm{MJ}$ and for hybrid fission-fusion pellets somewhat less energy would be here required. It is therefore conceivable that two beams each having an energy of $\sim 100 \mathrm{MJ}$ would suffice.

Second, the use of many beams approximating one beam of spherical symmetry and which are projected simultaneously into a spherical combustion chamber as shown in Fig. 2. There, after having entered the chamber, the different beam pulses would coalesce into one ion pulse having the shape of a spherical shell, the thickness of which would decrease with time as the pulse approaches the centre of convergence at which the pellet to be ignited is placed. For reactor conditions a total beam energy of $\sim 1 \mathrm{MJ}$ would be required, as in the case of laser fusion, but since the focusing here may not be as perfect as for optical radiation a larger input energy may be needed; this does not, however, seem to present a problem since the energy can be drawn from cheap inductive storage devices. The pulse length required for solid pellet heating is of the order $\sim 10^{-9} \mathrm{~s}$ and less. If the pellet has the form of a spherical shell the pulse can be somewhat longer. So if the pulse is delivered from the diode in about $\sim 10^{-6} \mathrm{~s}$ it has to be compressed by a factor $\sim 10^{3}$, shortening the pulse length down to $\sim 10^{-9} \mathrm{~s}$. This implies a radial beam compression from an initial beam width of $\sim 1 \mathrm{~m}$ down to $\sim 1 \mathrm{~mm}$. It seems that there is a fairly good chance that this can be done.

Desert Research Institute,

University of Nevada System,

Reno, Nevada 89507

Received April 22, revised June 20, 1974.

1 Nuckolls, J., et al., Nature, 239, 139 (1972).

2 Winterberg, F., Nature, 241, 449 (1973).

3 Winterberg, F., Phys. Rev., 174, 212 (1968).

4 Winterberg, F., in Physics of High Energy Density, 370 (Academic Press, New York, 1971).

5 Sudan, R. N., and Lovelace, R. V., Phys. Rev. Lett., 31, 1174 (1973).

\section{Measurement of the speed of light}

WE report here the completion of a determination of the speed of light at the National Physical Laboratory ${ }^{1}$. The value was obtained from the product of the measured frequency and the wavelength, determined through up-conversion, of the radiation from a $\mathrm{CO}_{2}$ laser stabilised to the $\mathrm{R}(12)$ transition of $\mathrm{CO}_{2}$ at $9.3 \mu \mathrm{m}$.

The frequency was determined relative to the caesium standard, using successive stages of harmonic multiplication and beat-frequency detection, with $\mathrm{HCN}$ and $\mathrm{H}_{2} \mathrm{O}$ lasers used as transfer oscillators. The value obtained ${ }^{2}$ was $v=$ $32,176,079,482 \mathrm{kHz}$, with a statistical uncertainty, $\sigma_{m}$, of
$0.13 \times 10^{-9}$, and a systematic uncertainty of $\pm 0.7 \times 10^{-9}$. (The systematic uncertainty is less than that published previously, because only the reproducibility of the $\mathrm{CO}_{2}$ stabilised frequency is considered, rather than the uncertainty of obtaining the unperturbed $R(12)$ transition frequency.) The wavelength was determined using interferometric measurements on upconverted light at $0.68 \mu \mathrm{m}$ and was related to the ${ }^{86} \mathrm{Kr}$ primary length standard, realised in accordance with the latest recommendations of the Comité Consultatif pour la Définition du Mètre (CCDM). The value obtained ${ }^{3}$ for the free space vacuum wavelength is $\lambda=9,317,246.348 \mathrm{pm}$, with $\sigma_{m}=1.14 \times 10^{-9}$ and a systematic uncertainty of $\pm 1.4 \times 10^{-9}$.

The product of these results gives the value for the speed of light

$$
c=299,792,459.0 \pm 0.8 \mathrm{~m} \mathrm{~s}^{-1}
$$

The uncertainty (corresponding to $\pm 2.7 \times 10^{-9}$ ) is the arithmetic sum of the standard error of the mean $\left(1.1 \times 10^{-9}\right)$ and the total systematic uncertainty $\left( \pm 1.6 \times 10^{-9}\right)$. Each of these was derived by quadrature summation of the contributions from the frequency and wavelength measurements.

Our measurement may be compared with values derived from a frequency measurement, by Evenson et al. ${ }^{4}$, of the methane stabilised $\mathrm{He}-\mathrm{Ne}$ laser at $3.39 \mu \mathrm{m}$, and the wavelength measurements made in that and other laboratories. The available data was reviewed by the CCDM in June 1973, when the method of realising the metre through the ${ }^{86} \mathrm{Kr}$ source was reconsidered. It was decided that where asymmetry of the spectral profile is observed, the defined wavelength applies to a point midway between the peak and centre of gravity. Thus, the result of Evenson et al. becomes: $c=299,792,457.4 \pm 1.1 \mathrm{~m} \mathrm{~s}^{-1}$. On consideration of wavelength measurements in four laboratories, however, the CCDM recommended ${ }^{5}$ for general use, the value $c=299,792,458 \mathrm{~m} \mathrm{~s}^{-1}$, with an uncertainty of $\pm 4 \times 10^{-9}$ resulting from the uncertainties of the wavelength measurements.

The satisfactory agreement of our result with those based upon the $3.39 \mu \mathrm{m}$ laser confirms both the recommended value for $c$ and the reliability of frequency and wavelength measurement techniques involving infrared laser radiations.
T. G. Blaney
C. C. BRADleY
G. J. EDWARDS
B. W. JOLLIFFE
D. J. E. KNIGHT
W. R. C. ROWLEY
K. C. SHOTTON
P. T. WOODS

National Physical Laboratory,

Teddington, Middlesex, UK

Received May 17; revised July 2, 1974.

${ }^{1}$ Bradley, C. C., Edwards, G. J., Knight, D. J. E., Rowley, W. R. C., and Woods, P. T., Phys. Bull., 23, 15 (1972).

2 Blaney, T. G., et al. Nature, 244, 504 (1973).

3 Jolliffe, B. W., Rowley, W. R. C., Shotton, K. C., Wallard, A. J., and Woods, P. T., Nature, 251, 46 (1974).

4 Evenson, K. M., et al., Phys. Rev. Lett., 29, 1346 (1972).

5 Terrien, J., Nouv. Revue Opt. 4, 215 (1973).

\section{Accurate wavelength measurement on up-converted $\mathrm{CO}_{2}$ laser radiation}

WAVELENGTH measurements, which are part of an accurate determination of the speed of light ${ }^{1}$, have been made on the radiation from a carbon dioxide laser, stabilised to the $R(12)$ transition at $9.3 \mu \mathrm{m}$ by saturated fluorescence in an external $\mathrm{CO}_{2}$ cell, which has a measured frequency ${ }^{2}$ reproducible to better than one part in $10^{9}$. The problems of a direct intercomparison of infrared and visible wavelengths were avoided 